\title{
MICROSCOPIC MODEL OF PHASE TRANSITION IN THE CRYSTALS OF THE DMAAIS AND DMAGaS TYPES
}

\author{
I. V. Stasyuk, O. V. Velychko \\ Institute for Condensed Matter Physics of the National Academy of Sciences of Ukraine, \\ 1 Svientsitskii Str., Lviv, UA-79011, Ukraine
}

(Received 10 December, 1999)

\begin{abstract}
The four-state model is proposed for the description of phase transition in ferroelectric crystals of the DMAGaS and DMAAlS type. Thermodynamic functions of the model are obtained in the mean field approximation. The phase transition between paraelectric and ferroelectric phases is investigated. It is established that the order of the phase transitions depends on the relations between model parameters.
\end{abstract}

Key words DMAAIS, DMAGaS, ferroelectrics, phase transitions, quantum statistical model.

PACS number(s): 77.84.-s, 64.60.Cn

\section{INTRODUCTION}

A peculiar feature of isomorphous crystals with ferroelectric properties $\left(\mathrm{CH}_{3}\right)_{2} \mathrm{NH}_{2} \mathrm{Al}\left(\mathrm{SO}_{4}\right)_{2} \cdot 6 \mathrm{H}_{2} \mathrm{O}$ (DMAAlS) and $\left(\mathrm{CH}_{3}\right)_{2} \mathrm{NH}_{2} \mathrm{Ga}\left(\mathrm{SO}_{4}\right)_{2} \cdot 6 \mathrm{H}_{2} \mathrm{O}$ (DMA$\mathrm{GaS})$ is the possibility of their existence in three different phases at a change of temperature: at room temperature the crystal is paraelectric, at the lowering of temperature it sequentially becomes ferroelectric and further can be in the antiferroelectric state [1-4].

It has been measured that $T_{c 1}=136 \mathrm{~K}$ and $T_{c 2}=$ $113 \mathrm{~K}$ [4] (or $122 \mathrm{~K}$ and $114 \mathrm{~K}$ respectively [3]) for the DMAGaS crystal, but it has been only found that $T_{c 1}=150 \mathrm{~K}[1-3]$ for DMAAlS. The phase transition between the ferroelectric and antiferroelectric phases is of the first order $[3,4]$. The phase transition paraelectric ferroelectric is of the first order close to the second one in DMAGaS and of the second order in DMAAlS. The crystallographic analysis shows that in all the three phases the crystal belongs to the monoclinic space groups: a high-temperature paraelectric phase has the $\mathrm{P} 2_{1} / \mathrm{n}$ space group $[1,2]$, the ferroelectric and antiferroelectric ones have $\mathrm{Pn}[2,3]$ and $\mathrm{P} 2_{1}$ [3] groups respectively. It should be mentioned that low-symmetry space groups are subgroups of the high-symmetry group obtained by the loss of rotation axis and mirror plane, respectively (point symmetry group $2 / \mathrm{m}$ changes to $\mathrm{m}$ or 2 ).

The data of the investigation of various physical properties of the crystals were presented in a series of publications. First of all we should mention here dielectric, pyroelectric and dilatometric measurements [4-9,4,10,11], ultrasonic and NMR investigations [12-14], studies of relaxation dynamics and lattice dynamics $[15,16]$ by means of radiospectroscopy and Raman spectroscopy methods. Such issues as dielectric anomalies in the vicinity of phase transition points (mainly near $T_{c 1}$ ), clarification of the order of phase transition, dynamics of ionic groups and protons on hydrogen bonds in the wide range of temperatures [15,16], influence of external hydrostatic pressure [17-19] were considered. Temperatures of phase transitions were justified; according to the latest data
$T_{c 1}=136 \mathrm{~K}, T_{c 2}=117 \mathrm{~K}(\mathrm{DMAGaS})$ and $T_{c 1}=155 \mathrm{~K}$ (DMAAlS).

The DMAAIS and DMAGaS crystals belong to the type of ferroelectrics which have some structural elements whose reorientation leads to the polarisation of the crystal. In this case the element which can be reoriented is the dimethylammonium cation (more strictly $\mathrm{NH}_{2}$ group). Crystals contain two symmetrically nonequivalent DMA groups per unit cell (Fig. 1).

DMA can occupy four equilibrium positions which are related in pairs by the inversion centre forming a slightly deformed cross (Fig. 2). It is very likely that this asymmetry (and the corresponding difference of energies of interaction between groups in various positions) is responsible for such a complicated behaviour of the crystal. In the paraelectric phase the site in one pair is occupied with the probability of $40 \%$ and in another with the probability of $10 \%$ at $300 \mathrm{~K}$.

A possible role of reorientations of the DMA groups in phase transitions of the considered family of crystals was pointed out in a number of articles $[3,6,13,20]$. This assumption is directly confirmed by the data of structural investigations $[13,20]$. Performed recently the NMR measurements [13] have proved that reorientations and orderings of the DMA groups can be considered as an origin of ferroelectrics (F) and antiferroelectrics (AF) phase transitions while other changes in a lattice structure (rotations of $\mathrm{SO}_{4}$ groups, freezing of protons in certain positions on hydrogen bonds) are just accompanying phenomena.

So for a theoretical description of phase transitions to ferro- and antiferroelectric states in DMAAlS and DMAGaS crystals and the development of appropriate microscopic model one should take into account the reorientation of DMA groups and their influence on the state of the crystal. Such an approach is a major goal of the present paper. Our task is to formulate this type model, to introduce order parameters responsible for the appearance of low-symmetry phases, to calculate a thermodynamic potential proceeding from the model and to investigate thermodynamically the stable states. The transition from paraelectric to ferroelectric state and the 
dependence of the order of this transition on the system parameter values will be studied in more detail.

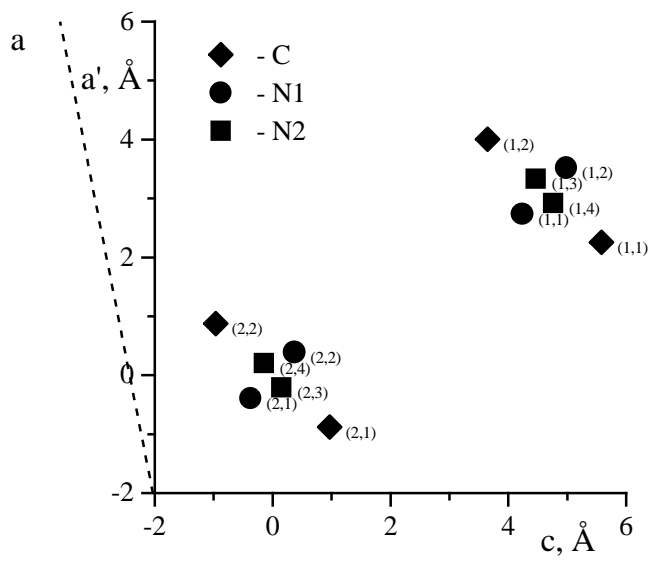

Fig. 1. Projection of $\mathrm{N}$ and $\mathrm{C}$ atoms in the dimethylammonium groups of DMAGaS crystal at ambient temperature [1] onto the $\mathrm{XZ}$ plane (position indices are indicated in parenthesis). The $\mathrm{Y}$ coordinates of atoms are as follows: $\mathrm{Y}_{N(1,1)}=-0.1469 \AA, \mathrm{Y}_{N(1,2)}=0.1469 \AA, \mathrm{Y}_{N(1,3)}=-0.6736 \AA$, $\mathrm{Y}_{N(1,4)}=0.6736 \AA, \mathrm{Y}_{N(2,1)}=5.5099 \AA, \mathrm{Y}_{N(2,2)}=5.2161 \AA$, $\mathrm{Y}_{N(2,3)}=4.6894 \AA, \mathrm{Y}_{N(2,4)}=6.0366 \AA, \mathrm{Y}_{C(1,1)}=-0.2606 \AA$, $\mathrm{Y}_{C(1,2)}=0.2606 \AA, \mathrm{Y}_{C(2,1)}=5.6236 \AA, \mathrm{Y}_{C(2,2)}=5.1024 \AA$.

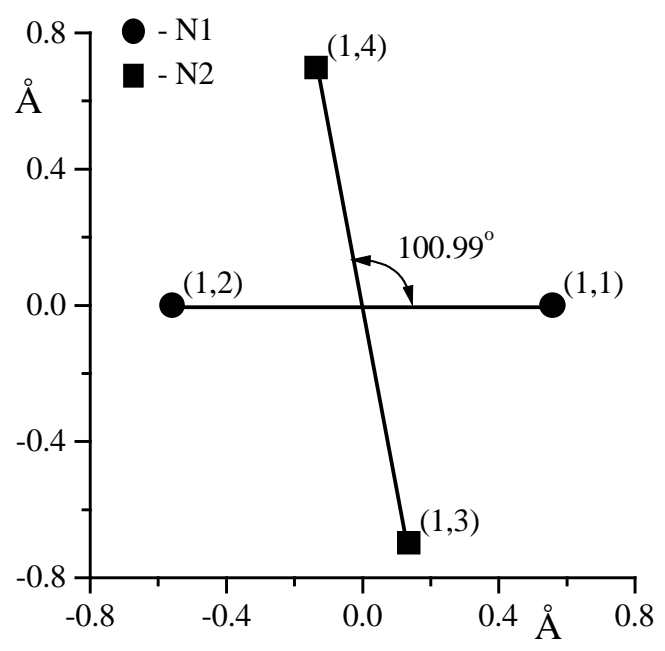

Fig. 2. Projection of $\mathrm{N}$ atoms onto the plane perpendicular to the $\mathrm{C}-\mathrm{C}^{\prime}$ axis of the dimethylammonium group.

\section{HAMILTONIAN OF THE MODEL}

In both high- and low-temperature phases ionic groups belong to two different sublattices (an elementary cell consists of two translationally nonequivalent groups). We shall characterize orientations of the groups by the spatial localization of nitrogen atoms belonging to the groups. Four possible positions of $N$ atom are described by the Hubbard projection operators $X_{n k}^{p p}$, where $n$ is the number of a lattice site (an elementary cell), $\alpha=1,2$ is the sublattice index, $p=1, \ldots, 4$ is the position number $\left(X_{n \alpha}^{p p}=0\right.$ or $\left.1 ; \sum_{p} X_{n \alpha}^{p p}=1\right)$. Energies of different positions are pairwise equivalent: $\varepsilon_{1}=\varepsilon_{2}, \varepsilon_{3}=\varepsilon_{4}$ (see notations in Figs. 1 and 2).

The model Hamiltonian can be written down as follows:

$$
H=\sum_{n \alpha p} \varepsilon_{p} X_{n \alpha}^{p p}-\frac{1}{2} \sum_{n n^{\prime}} \sum_{\substack{\alpha \alpha^{\prime} \\ p p^{\prime}}} J_{\alpha \alpha^{\prime}}^{p p^{\prime}}\left(n n^{\prime}\right) X_{n \alpha}^{p p} X_{n^{\prime} \alpha^{\prime}}^{p^{\prime} p^{\prime}}
$$

where the first and the second terms describe energy of separate complexes and pair interaction $J_{\alpha \alpha^{\prime}}^{p p^{\prime}}\left(n n^{\prime}\right)$ depending on their orientation states, respectively.

Further consideration will be performed in the mean field approximation. By substituting $X X \rightarrow 2\langle X\rangle X-$ $\langle X\rangle\langle X\rangle$ in (1) one can obtain

$$
H_{\mathrm{MF}}=N H_{c}+\sum_{n \alpha p}\left[\varepsilon_{p}-f_{\alpha p}\right] X_{n \alpha}^{p p}
$$

where the mean field is introduced

$$
f_{\alpha p}=\sum_{n^{\prime} \alpha^{\prime} p^{\prime}} J_{\alpha \alpha^{\prime}}^{p p^{\prime}}\left(n n^{\prime}\right)\left\langle X_{n^{\prime} \alpha^{\prime}}^{p^{\prime} p^{\prime}}\right\rangle
$$

and

$$
H_{\mathrm{c}}=\frac{1}{2} \sum_{n^{\prime}} \sum_{\substack{\alpha \alpha^{\prime} \\ p p^{\prime}}} J_{\alpha \alpha^{\prime}}^{p p^{\prime}}\left(n n^{\prime}\right)\left\langle X_{n \alpha}^{p p}\right\rangle\left\langle X_{n^{\prime} \alpha^{\prime}}^{p^{\prime} p^{\prime}}\right\rangle
$$

The averages $\left\langle X_{n \alpha}^{p p}\right\rangle$ do not depend on the cell number $\left(\left\langle X_{n \alpha}^{p p}\right\rangle \equiv\left\langle X_{\alpha}^{p p}\right\rangle\right)$ due to translational symmetry which does not change at the transition to low-symmetry phases (lattice period is not doubled in the antiferroelectric phase). The averages have the meaning of the occupation numbers of the corresponding orientation states and can be grouped in linear combinations

$$
\xi_{\mu}=\sum_{\alpha p} U_{\mu, \alpha p}\left\langle X_{\alpha}^{p p}\right\rangle
$$

which transform according to irreducible representations of the point symmetry group $2 / m$ of the hightemperature phase. Coefficients of transformations (5) are presented in Table 1 . As one can see all the possible irreducible representations $\left(\mathrm{A}_{g}, \mathrm{~B}_{g}, \mathrm{~B}_{u}, \mathrm{~A}_{u}\right)$ are realized, two linear combinations of the type of (5) belong to each of them.

Combinations $\xi_{\mu}$ which are transformed according to $B_{u}$ and $A_{u}$ representations are connected with the differences of occupying the $(\alpha, 1),(\alpha, 2)$ and $(\alpha, 3),(\alpha, 4)$ positions and can play a role of the order parameters for phase transitions to ferroelectric $\left(B_{u}\right)$ and antiferroelectric $\left(A_{u}\right)$ phases. 
As one can see in Table 1 the first of the parameters belonging to the $B_{u}$ representation $\left(y_{+}\right)$describes ferroelectric ordering along the ferroelectric axis (the OX' axis in the crystallographic plane (ac)) while the second one $\left(u_{-}\right)$corresponds to antiferroelectric ordering along OY (the crystallographic axis b). For the $A_{u}$ representation the ordering is inverse: the parameter $y_{-}$describes antiferroelectric ordering along OX' and the parameter $u_{+}$ defines ferroelectric ordering along OY. Thus, the states of ferro- and antiferrophase observed in DMAAlS and DMAGaS crystals in reality are mixed.

\begin{tabular}{|c|c||c|c|c|c|c|c|c|c|}
\hline & & $(1,1)$ & $(1,2)$ & $(1,3)$ & $(1,4)$ & $(2,1)$ & $(2,2)$ & $(2,3)$ & $(2,4)$ \\
\hline \hline \multirow{2}{*}{$\mathrm{A}_{g}$} & $x_{+}$ & $1 / 2$ & $1 / 2$ & 0 & 0 & $1 / 2$ & $1 / 2$ & 0 & 0 \\
\cline { 2 - 10 } & $z_{+}$ & 0 & 0 & $1 / 2$ & $1 / 2$ & 0 & 0 & $1 / 2$ & $1 / 2$ \\
\hline \multirow{2}{*}{$\mathrm{B}_{g}$} & $x_{-}$ & $1 / 2$ & $1 / 2$ & 0 & 0 & $-1 / 2$ & $-1 / 2$ & 0 & 0 \\
\cline { 2 - 9 } & $z_{-}$ & 0 & 0 & $1 / 2$ & $1 / 2$ & 0 & 0 & $-1 / 2$ & $-1 / 2$ \\
\hline \multirow{2}{*}{$\mathrm{B}_{u}$} & $y_{+}$ & $1 / 2$ & $-1 / 2$ & 0 & 0 & $1 / 2$ & $-1 / 2$ & 0 & 0 \\
\cline { 2 - 9 } & $u_{-}$ & 0 & 0 & $1 / 2$ & $-1 / 2$ & 0 & 0 & $-1 / 2$ & $1 / 2$ \\
\hline \multirow{2}{*}{$\mathrm{A}_{u}$} & $y_{-}$ & $1 / 2$ & $-1 / 2$ & 0 & 0 & $-1 / 2$ & $1 / 2$ & 0 & 0 \\
\cline { 2 - 9 } & $u_{+}$ & 0 & 0 & $1 / 2$ & $-1 / 2$ & 0 & 0 & $1 / 2$ & $-1 / 2$ \\
\hline
\end{tabular}

Table 1. Coefficients of symmetrized occupancies of orientation states which correspond to irreducible representations of the point symmetry group $2 / \mathrm{m}$.

Using (3) and (5) one can write down

$$
f_{\alpha p}=\sum_{\mu \mu^{\prime}}\left(U^{-1}\right)_{\alpha p, \mu} \tilde{j}_{\mu \mu^{\prime}} \xi_{\mu^{\prime}}
$$

where

$$
\begin{aligned}
\tilde{j}_{\mu \mu^{\prime}} & =\sum_{\alpha p} \sum_{\alpha^{\prime} p^{\prime}} U_{\mu, \alpha p} j_{\alpha \alpha^{\prime}}^{p p^{\prime}}\left(U^{-1}\right)_{\alpha^{\prime} p^{\prime}, \mu^{\prime}}, \\
j_{\alpha \alpha^{\prime}}^{p p^{\prime}} & =\sum_{n^{\prime}} J_{\alpha \alpha^{\prime}}^{p p^{\prime}}\left(n n^{\prime}\right)
\end{aligned}
$$

and

$$
H_{c}=\frac{1}{2} \sum_{\mu \mu^{\prime}} \tilde{j}_{\mu \mu^{\prime}} \xi_{\mu} \xi_{\mu^{\prime}}
$$

\section{FREE ENERGY AND EQUATIONS FOR ORDER PARAMETERS}

Proceeding from expressions (2), (6) and (8) one can easily derive the main thermodynamic functions of the model in the mean field approximation and construct a set of equations for the selfconsistency parameters $\xi_{\mu}$.

The corresponding partition function is

$$
Z=\mathrm{e}^{-\beta N H_{c}}\left[\sum_{p=1}^{4} \mathrm{e}^{-\beta\left(\varepsilon_{p}-f_{1 p}\right)}\right]^{N}\left[\sum_{p=1}^{4} \mathrm{e}^{-\beta\left(\varepsilon_{p}-f_{2 p}\right)}\right]^{N}
$$

or

$$
Z=\mathrm{e}^{-\beta N H_{c}}\left[2 Z_{1}\right]^{N}\left[2 Z_{2}\right]^{N},
$$

where

$$
\begin{aligned}
& Z_{1}=Q_{x}^{+} \cosh \beta\left(k_{y+}+k_{y-}\right)+Q_{z}^{+} \cosh \beta\left(k_{u-}+k_{u+}\right), \\
& Z_{2}=Q_{x}^{-} \cosh \beta\left(k_{y+}-k_{y-}\right)+Q_{z}^{-} \cosh \beta\left(k_{u_{-}}-k_{u+}\right),
\end{aligned}
$$

and

$$
\begin{array}{ll}
Q_{x}^{ \pm}=\mathrm{e}^{-\beta \varepsilon_{1}} \exp \left[-\beta\left(k_{x+} \pm k_{x-}\right)\right], \quad Q_{z}^{ \pm}=\mathrm{e}^{-\beta \varepsilon_{3}} \exp \left[-\beta\left(k_{z+} \pm k_{z-}\right)\right], \\
k_{x+}=\left(A_{1} x_{+}+C_{1} z_{+}\right) / 2, \quad k_{x-}=\left(A_{2} x_{-}+C_{2} z_{-}\right) / 2, \\
k_{z+}=\left(C_{1} x_{+}+B_{1} z_{+}\right) / 2, \quad k_{z-}=\left(C_{2} x_{-}+B_{2} z_{-}\right) / 2, \\
k_{y+}=\left(A_{3} y_{+}+C_{3} u_{-}\right) / 2, \quad k_{y-}=\left(A_{4} y_{-}+C_{4} u_{+}\right) / 2, \\
k_{u-}=\left(C_{3} y_{+}+B_{3} u_{-}\right) / 2, \quad k_{u+}=\left(C_{4} y_{-}+B_{4} u_{+}\right) / 2 .
\end{array}
$$

Here such notations are introduced

$$
\begin{aligned}
& A_{1}=a+b+g+h, B_{1}=e+f+l+m, C_{1}=c+d+j+k, \\
& A_{2}=a+b-g-h, B_{2}=e+f-l-m, C_{2}=c+d-j-k,
\end{aligned}
$$




$$
\begin{aligned}
& A_{3}=a-b+g-h, B_{3}=e-f-l+m, C_{3}=c-d-j+k, \\
& A_{4}=a-b-g+h, B_{4}=e-f+l-m, C_{4}=c-d+j-k, \\
& a=j_{11}^{11}(0), b=j_{11}^{12}(0), c=j_{11}^{13}(0), d=j_{11}^{14}(0), \\
& e=j_{11}^{33}(0), f=j_{11}^{34}(0), g=j_{12}^{11}(0), h=j_{12}^{12}(0), \\
& j=j_{12}^{13}(0), k=j_{12}^{14}(0), l=j_{12}^{33}(0), m=j_{12}^{34}(0) .
\end{aligned}
$$

Free energy per site $F$ is

$$
F=H_{c}-\Theta \ln \left(2 Z_{1}\right)-\Theta \ln \left(2 Z_{2}\right) .
$$

By direct averaging or with the use of free energy (16) one can obtain the set of equations for the parameters $\xi_{\mu}$ :

$$
\begin{aligned}
& x_{ \pm}=\left[Z_{1}^{-1} Q_{x}^{+} \cosh \beta\left(k_{y+}+k_{y-}\right) \pm Z_{2}^{-1} Q_{x}^{-} \cosh \beta\left(k_{y+}-k_{y-}\right)\right] / 2, \\
& z_{ \pm}=\left[Z_{1}^{-1} Q_{z}^{+} \cosh \beta\left(k_{u+}+k_{u-}\right) \pm Z_{2}^{-1} Q_{z}^{-} \cosh \beta\left(k_{u+}-k_{u-}\right)\right] / 2, \\
& y_{ \pm}=\left[Z_{1}^{-1} Q_{x}^{+} \sinh \beta\left(k_{y+}+k_{y-}\right) \pm Z_{2}^{-1} Q_{x}^{-} \sinh \beta\left(k_{y+}-k_{y-}\right)\right] / 2, \\
& u_{ \pm}=\left[Z_{1}^{-1} Q_{z}^{+} \sinh \beta\left(k_{u+}+k_{u-}\right) \pm Z_{2}^{-1} Q_{z}^{-} \sinh \beta\left(k_{u+}-k_{u-}\right)\right] / 2 .
\end{aligned}
$$

\section{PHASE TRANSITION BETWEEN PARAELECTRIC AND FERROELECTRIC PHASES}

Let us consider phase transition (PT) from the paraelectric phase to the ferroelectric one which corresponds to the irreducible representation $B_{u}$ with the two-component order parameter (OP). In this case the averages $x_{-}, z_{-}, y_{-}$ and $u_{+}$are equal to zero, the averages $y_{+}$and $u_{-}$are the components of $\mathrm{OP}$ and $Z_{1}=Z_{2}$. Then the expression for free energy (16) simplifies

$$
\begin{aligned}
F & =H_{c}-2 \Theta \ln \left(2 Z_{1}\right), \\
H_{c} & =\frac{1}{2}\left(A_{1} N_{1}^{2}+2 C_{1} N_{1} N_{2}+B_{1} N_{2}^{2}+A_{3} \xi^{2}+2 C_{3} \xi \eta+B_{3} \eta^{2}\right), \\
Z_{1} & =\mathrm{e}^{-\beta \varepsilon_{1}} \exp \left[-\frac{1}{2} \beta\left(A_{1} N_{1}+C_{1} N_{2}\right)\right] \cosh \left[\frac{1}{2} \beta\left(A_{3} \xi+C_{3} \eta\right)\right] \\
& +\mathrm{e}^{-\beta \varepsilon_{3}} \exp \left[-\frac{1}{2} \beta\left(C_{1} N_{1}+B_{1} N_{2}\right)\right] \cosh \left[\frac{1}{2} \beta\left(C_{3} \xi+B_{3} \eta\right)\right] .
\end{aligned}
$$

Here

$$
N_{1}=x_{+}, N_{2}=z_{+}, \xi=y_{+}, \eta=u_{-} .
$$

It is convenient to separate the term in the expression for the free energy which depends on $\xi$ and $\eta$

$$
\begin{aligned}
F & =F_{0}+F_{1}(\xi, \eta), \\
F_{0} & =\frac{1}{2}\left(A_{1} N_{1}^{2}+2 C_{1} N_{1} N_{2}+B_{1} N_{2}^{2}\right)-2 \Theta \ln 2-2 \Theta \ln (a+b), \\
F_{1}(\xi, \eta) & =\left(A_{3} \xi^{2}+2 C_{3} \xi \eta+B_{3} \eta^{2}\right)
\end{aligned}
$$




$$
-2 \Theta \ln \left\{n_{1} \cosh \left[\frac{1}{2} \beta\left(A_{3} \xi+C_{3} \eta\right)\right]+n_{2} \cosh \left[\frac{1}{2} \beta\left(C_{3} \xi+B_{3} \eta\right)\right]\right\}
$$

where the following symbols are introduced

$$
\begin{aligned}
& a=\mathrm{e}^{-\beta \varepsilon_{1}} \exp \left[-\frac{1}{2} \beta\left(A_{1} N_{1}+C_{1} N_{2}\right)\right], b=\mathrm{e}^{-\beta \varepsilon_{3}} \exp \left[-\frac{1}{2} \beta\left(C_{1} N_{1}+B_{1} N_{2}\right)\right], \\
& n_{1}=a /(a+b), n_{2}=b /(a+b), n_{1}=1-n_{2} .
\end{aligned}
$$

Expressions $n_{1}$ and $n_{2}$ describe an effective occupancy of the equilibrium positions 1,2 and 3,4 , respectively, in a disordered state. One can assume they have a weak dependence on temperature in the region of the paraelectricferroelectric phase transition. In the framework of this approach $n_{1}$ and $n_{2}$ could be considered as model parameters. The main contribution to the change of free energy is given by the term $F_{1}(\xi, \eta)$. Then the averages $\xi$ and $\eta$ are given by a set of equations

$$
\begin{aligned}
\xi & =\frac{(1-n) \sinh [\tilde{\beta}(\xi+C \eta)]}{(1-n) \cosh [\tilde{\beta}(\xi+C \eta)]+n \cosh [\tilde{\beta}(C \xi+B \eta)]} \\
\eta & =\frac{n \sinh [\tilde{\beta}(C \xi+B \eta)]}{(1-n) \cosh [\tilde{\beta}(\xi+C \eta)]+n \cosh [\tilde{\beta}(C \xi+B \eta)]}
\end{aligned}
$$

where the following symbols are used for normalized parameters

$$
\begin{aligned}
& B=B_{3} / A_{3}, C=C_{3} / A_{3}, \\
& \tilde{\beta}=\beta A_{3} / 2, n=n_{2} .
\end{aligned}
$$

The set of equations (23) is invariant with respect to the inversion operation $\xi, \eta \rightarrow-\xi,-\eta$ and has, as usual, a trivial paraelectric solution $\xi=\eta=0$. It can be established that at $\Theta=0$ two other solutions $(\xi=0, \eta=1$ at $C<B$ and $\xi=1, \eta=0$ at $C<1)$ exist.

The Landau expansion of the free energy is a convenient tool for investigating PTs of the second order (PT2) and of the first order (PT1) which are close to the second one. But in this case the use of the Landau expansion is connected with problems due to the negative sign of the coefficient at the sixth order of $\mathrm{OP}$ for the same range of parameter values. For this reason the Landau expansion plays an auxiliary role in our investigations. Besides that we perform all the calculations of $\xi$ and $\eta$ averages, free energy and the temperature of PT numerically using expression (20) and set (23).

The Landau expansion up to the fourth order was used for calculating the temperature of possible PT2 and boundaries of the regions where PT1 can occur. In this case $\mathrm{OP}$ is two-component, hence for diagonalization of the quadratic form, which looks like

$$
F_{1}(\xi, \eta)=U \xi^{2}+V \eta^{2}+2 S \xi \eta
$$

$$
\begin{aligned}
& U=\frac{1}{2}\left[1-\left(1+\left(C^{2}-1\right) n\right) \frac{\tilde{\beta}}{2}\right], \\
& V=\frac{1}{2}\left[B-\left(C^{2}+\left(B^{2}-C^{2}\right) n\right) \frac{\tilde{\beta}}{2}\right], \\
& S=\frac{C}{2}\left[1-(1+(B-1) n) \frac{\tilde{\beta}}{2}\right]
\end{aligned}
$$

one should make the following transformation

$$
\begin{aligned}
& \left(\begin{array}{l}
r_{1} \\
r_{2}
\end{array}\right)=\hat{T}\left(\begin{array}{l}
\xi \\
\eta
\end{array}\right), \quad \hat{T}=\left(\begin{array}{cc}
\cos \varphi & -\sin \varphi \\
\sin \varphi & \cos \varphi
\end{array}\right), \\
& \cos 2 \varphi=(V-U) / \sqrt{(V-U)^{2}+4 S^{2}} \\
& \sin 2 \varphi=2 S / \sqrt{(V-U)^{2}+4 S^{2}}
\end{aligned}
$$

Now expression (24) is as follows

$$
\begin{aligned}
F_{2}\left(r_{1}, r_{2}\right) & =\Lambda_{1} r_{1}^{2}+\Lambda_{2} r_{2}^{2}, \\
\Lambda_{1} & =\frac{1}{2}\left[(V+U)-\sqrt{(V-U)^{2}+4 S^{2}}\right], \\
\Lambda_{2} & =\frac{1}{2}\left[(V+U)+\sqrt{(V-U)^{2}+4 S^{2}}\right] .
\end{aligned}
$$

Coefficients $\Lambda_{1}$ and $\Lambda_{2}$ equal zero at the temperatures 


$$
\tilde{\Theta}_{1,2}=\left[\left(B-C^{2}\right)(1-n) n\right] /\left[(1-(1-B) n) \mp \sqrt{(1-(1+B) n)^{2}+4 C^{2}(1-n) n}\right] .
$$

One can obtain the conditions on parameters $A_{3}, B_{3}$ and $C_{3}$

$$
A_{3}>0, B_{3}>0, A_{3} B_{3}>C_{3} ; \quad B>0, B>C^{2},
$$

taking into account that at $\Theta=\infty(\beta=0)$ the system is paraelectric (coefficients $\Lambda_{1}$ and $\Lambda_{2}$ are positive). If the conditions (30) are satisfied, the temperature $\tilde{\Theta}_{1}$ is always higher than the temperature $\tilde{\Theta}_{2}$. Hence it determines that the temperature of possible PT2 and variable $r_{1}$ in expression (28) becomes the OP.

The region of parameter values, where PT1 takes place, can be established by the criterion of a negative fourth order term proportional to $r_{1}^{4}$ of the Landau expansion. This term can be obtained from the expression

$$
\begin{aligned}
F_{1}^{(4)}(\xi, \eta) & =-\frac{\tilde{\beta}}{32}\left\{\left(2 k_{1}+2 k_{2} C^{4}-(1-n) n C^{2}\right) \xi^{4}+\left(2 k_{1} C^{4}+2 k_{2} B^{4}-(1-n) n B^{2} C^{2}\right) \eta^{4}\right. \\
& +2 C\left(4 k_{1}+4 k_{2} B C^{2}-(1-n) n\left(B+C^{2}\right)\right) \xi^{3} \eta+2 C\left(4 k_{1} C^{2}+4 k_{2} B^{3}-(1-n) n B\left(B+C^{2}\right)\right) \xi \eta^{3} \\
& \left.+\left(12\left(k_{1}+k_{2} B^{2}\right) C^{2}-(1-n) n C^{2}\left(B^{2}+4 B C^{2}+C^{4}\right)\right) \xi^{2} \eta^{2}\right\} \\
k_{1} & =(1-n) / 12-(1-n)^{2} / 4, k_{2}=n / 12-n^{2} / 4
\end{aligned}
$$

using transformation (26).

It is convenient to make a qualitative analysis of the PT2 region in the case of $C=0$, when free energy (24) has a diagonal form and OP is equal to $\xi$ or $\eta$. The equation $B=1 / n-1$ describes the curve which separates the regions with different $\mathrm{OP}$ on the $B-n$ diagram. This equation can be obtained by setting equal the temperatures at which coefficients at $\xi$ and $\eta$ in the quadratic form (24) are equal to zero. It is found that the sign of the coefficient at the fourth order of OP depends on $n$ only. The obtained diagram of a PT order is depicted in Fig. 3.

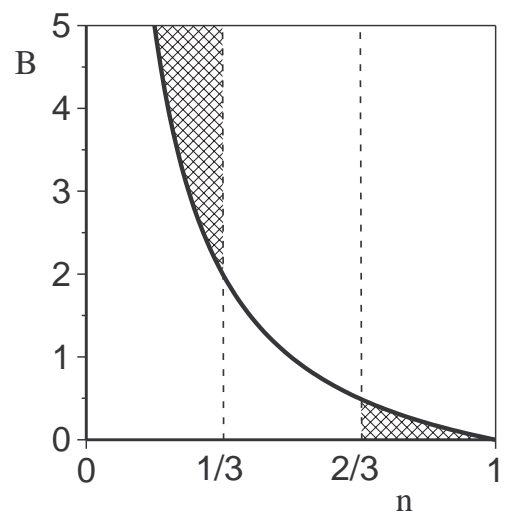

Fig. 3. Diagram of the order of the paraelectricferroelectric phase transition at $C=0$. Filled regions correspond to PT1, blank regions - PT2.
An increase of $C$ leads to the narrowing of the region of $\mathrm{n}$ where PT1 is possible (Fig. 4). One can see that the region, determined by the $F_{1}^{(4)}$ sign change, is smaller than obtained by direct numerical calculation. This difference can be explained on the example of the case $C=0$. The point $n^{\prime}$ is situated on the OP $\xi \rightarrow \eta$ change curve of the diagram in Fig. 3. On the left of the $n^{\prime}$ point the OP is $\xi$, hence PT2 on $\xi$ should take place before PT2 in $\eta$. But actually PT1 in $\eta$ takes place before them. It is illustrated in Fig. 5 where dependences of free energy and parameters $\xi, \eta$ on temperature are depicted. It is also possible to find PT2 in $\xi$ at first and PT1 with a jump of $\eta$ at a lower temperature. One should note that at $C \neq 0$ the OP $r_{1}$ is a mixture of $\xi$ and $\eta$. But at the considered values of parameters the main contribution to the jump is given by $\eta$. The diagram in Fig. 3 shows that PT1 with the prevailing jump of $\xi$ is typical for the region of small values of $B$ and large $n$; with the prevailing jump of $\eta$ - for large $B$ and small $n$. An increase of $C$ causes a stronger "mixing" of $\xi$ and $\eta$, but there is the limit $C^{2}<B(30)$ of the value of $C$. Thus it seems difficult to find in this approach the PT1 with the prevailing jump of $\xi$ (i.e. with the prevailing occupancies of positions 1 and 2) at small values of $n$ that is characteristic of DMAGaS crystals at PT from the paraelectric phase into the ferroelectric one. At small values of $n$ the model exhibits PT2 (close to PT1) with the prevailing change of the $\xi$ parameter (Fig. 6). So, we see that despite the simplicity of the model its thermodynamics is very complicated. As an example an exotic case with sophisticated dependencies of $\xi$ and $\eta$ on temperature is shown in Fig. 7 . 


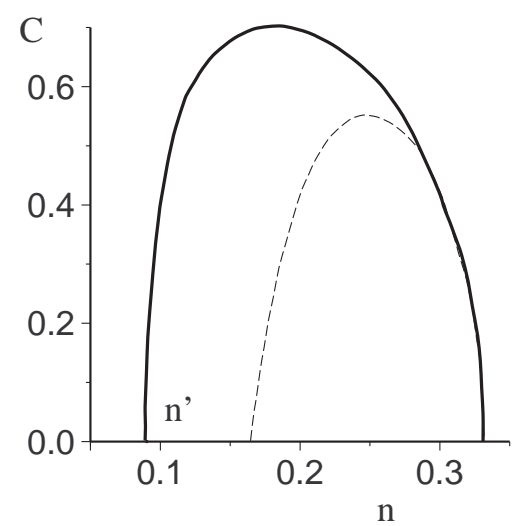

Fig. 4. Boundaries of PT1 region (inside the curves) at $B=5$. Solid line corresponds to numerical calculations, dashed one to the result of the Landau expansion analysis.

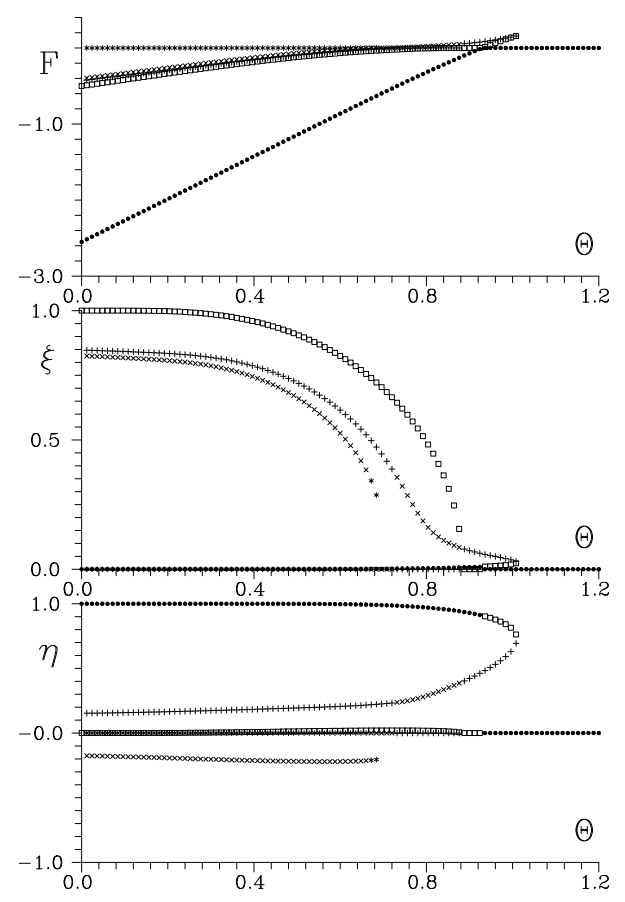

Fig. 5. Dependences of free energy and order parameters on temperature at the values of system parameters as $B=5$, $C=0.1, n=0.12$.

\section{CONCLUSIONS}

A microscopic approach based on the allowance for different orientation states of DMA ionic groups in crystal lattice is proposed in the present work for the description of phase transition in DMAAIS and DMAGaS crystals. In the framework of a simple four-state model order parameters are introduced and calculations of thermodynamic characteristics are made. The phase transition from the paraelectric to the ferroelectric state is studied at different relations between parameters describing the interactions of orientation states of different ionic groups.
An interesting feature which follows from the symmetry analysis is the strict correlation between the ordering along the 1-2 position axis (the ferroelectric one) and the $3-4$ position axis. Namely, as follows from Table 1, ferroelectric ordering along the 1-2 axis is accompanied by the antiferroelectric one along the $3-4$ axis and vice versa.

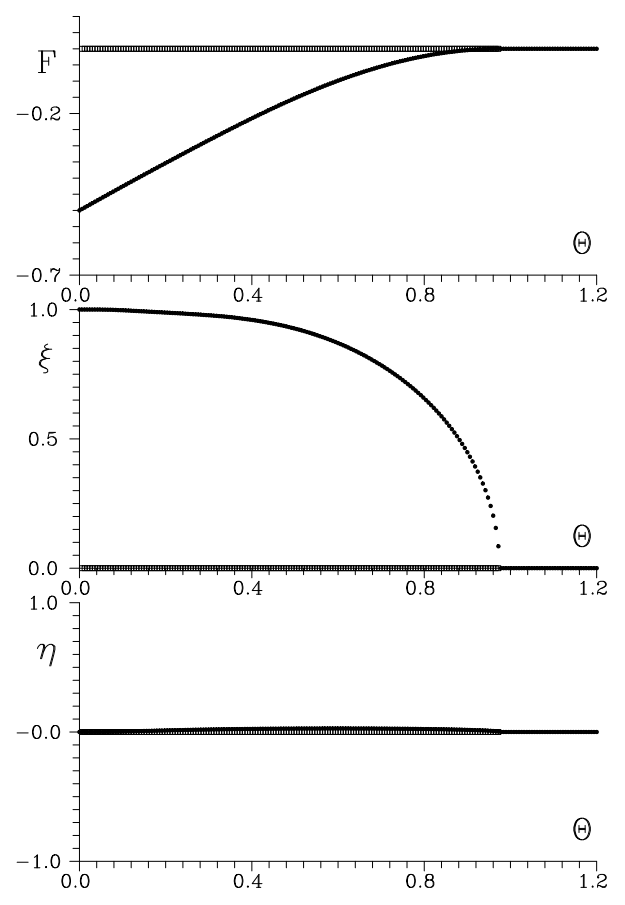

Fig. 6. Dependences of free energy and order parameters on temperature at the values of system parameters as $B=0.5, C=0.7, n=0.05$.

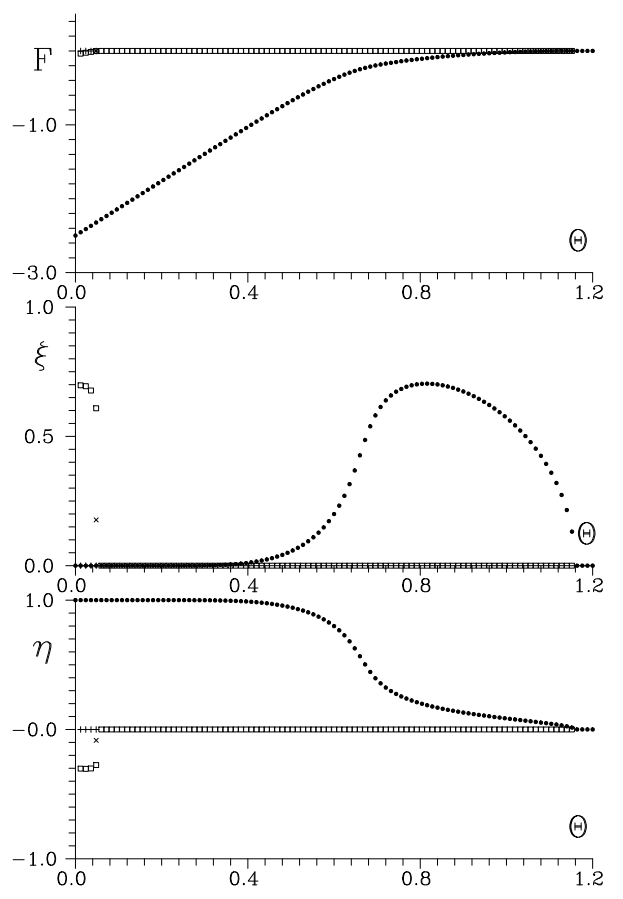

Fig. 7. Dependences of free energy and order parameters on temperature at the values of system parameters as $B=5$, $C=2, n=0.05$ 
The ferroelectric state in these crystals is simultaneously the improper antiferroelectric one along the other axis and the antiferroelectric state is accompanied by ferroelectric ordering along the mentioned axis. The factors affecting the order of the phase transition at $T_{c 1}$ are studied in more details on the basis of the model. At small values of $n$ this phase transition is established to be of the second order close to the first order (close to the tricritical point) under the approximation that total occupancies of positions $(1,2)$ and $(3,4)$ are assumed to be the same as in disordered phase and temperature independent. This behaviour coincides with the known result for the DMAAlS crystal but does not match with the data observed in DMAGaS crystals. For the latter case it should be necessary to abandon the approximation made and explicitly take into account the temper- ature dependences of the values of $n_{1}$ and $n_{2}$ with the use of expressions (22). Such an approach should also be adopted for the description of the low-temperature transition (at $T_{c 2}$ ) to the antiferroelectric phase and a study of the conditions of its realization. This can be the closest prospect of developing the model.

\section{ACKNOWLEDGEMENTS}

The authors would like to thank Prof. Z. Czapla and Dr. R. Tchukwinskyi for their interest in the work and useful discussions.

This work was supported in part by the Foundation for Fundamental Investigations of Ukrainian Ministry in the Affairs of Science and Technology, project No. 2.4/171.
[1] A. Pietraszko, K. Łukaszewicz, L. F. Kirpichnikova, Polish J. Chem. 67, 1877 (1993).

[2] A. Pietraszko, K. Eukaszewicz, Polish J. Chem. 68, 1239 (1994).

[3] A. Pietraszko, K. Łukaszewicz, L. F. Kirpichnikova, Polish J. Chem. 69, 922 (1995).

[4] R. Tchukvinskyi, R. Cach, Z. Czapla, Z. Naturforsch. Teil A 53, 105 (1998).

[5] E. F. Andreev, V. M. Varikash, L. A. Shuvalov, Izv. Akad. Nauk SSSR, Ser. Fiz. 53, 572 (1999).

[6] P. Sobiestinskas, Y. Grigas, E. F. Andreev, E. M. Varikash, Phase Transitions 40, 85 (1992).

[7] V. Kapustianik, M. Bublyk, I. Polovinko, S. Sveleba, Z. Trybula, E. Andreev, Phase Transitions 49, 231 (1994).

[8] S. Dacko, Z. Czapla, Ferroelectrics 185, 143 (1996).

[9] H. Pykacz, Z. Czapla, Ferroelectrics Lett. 22, 107 (1997).

[10] Z. Czapla, R. Tchukvinskyj, Acta Phys. Pol. A 93, 527 (1998).

[11] D. Podsiadła, Z. Czapla, B. Andrievsky, O. Myshchy- shyn, Acta Phys. Pol. A 96, 409 (1999).

[12] J. Furtak, Z. Czapla, Ferroelectrics Lett. 23, 63 (1997).

[13] J. Dolinšek, M. Klanjšek, D. Arčon, Hae Jin Kim, J. Selinger, V. Z̆agar, Phys. Rev. B 59, 3460 (1999).

[14] N. Alsabbagh, D. Michel, J. Furtak, Z. Czapla, Phys. Status Solidi A 167, 77 (1998).

[15] W. Bednarski, S. Waplak, L. F. Kirpichnikova, L. A. Shuvalov, Phys. Status Solidi A 160, R1 (1997).

[16] V. I. Torgashev, Yu. I. Yuzyuk, L. F. Kirpichnikova, L. A. Shuvalov, E. F. Andreev, Kristallografiya 36, 677 (1991).

[17] N. Yasuda, A. Kaneda, Z. Czapla, Ferroelectrics 223, 71 (1999).

[18] N. Yasuda, H. Tajima, Z. Czapla, Phys. Lett. A 192, 137 (1994).

[19] N. Yasuda, H. Tajima, Z. Czapla, J. Korean Phys. Soc. 32, S283 (1998).

[20] V. Yu. Kazimirov, E. E. Rieder, V. A. Sarin, A. V. Belushkin, L. A. Shuvalov, L. E. Fykin, C. Ritter, J. Korean Phys. Soc. 32, S91 (1998).

\title{
МІКРОСКОПІЧНА МОДЕЛЬ ФАЗОВИХ ПЕРЕХОДІВ У КРИСТАЛАХ ТИПУ DMAAIS TA DMAGaS
}

\author{
I. В. Стасюк, О. В. Величко \\ Інститут фізики конденсованих систем НАН Украйни, \\ вул. Свениіиького, 1, Львів, 79011, Украйна
}

\footnotetext{
Запропоновано чотиристанову модель для опису послідовности фазових переходів у сегнетоелектричних кристалах типу DMAGaS i DMAAIS. У наближенні середнього поля отримано термодинамічні функпії моделі. Фазовий перехід між пара- та сегнетоелектричною фазами досліджено за допомогою розвинення Ландау та чисельних розрахунків. Установлено, що рід фазового переходу залежить від співвідношень між параметрами моделі.
} 